\title{
SUPERLUMINOUS DEVICES VERSUS LOW-LEVEL LASER FOR TEMPOROMANDIBULAR DISORDERS
}

\author{
V. Sveshtarov ${ }^{1}$, S. Nencheva-Sveshtarova ${ }^{2}$, R. Grozdanova ${ }^{2}$, K. Prodanova ${ }^{3}$ \\ 'Department Oral and Maxillofacial Surgery, Faculty of Dental Medicine, Medical University - Sofia, Bulgaria \\ ${ }^{2}$ Center for Integrated Dental Medicine, Faculty of Dental Medicine, Medical University - Sofia, Bulgaria \\ ${ }^{3}$ Department Stochastics and Optimization, Faculty of Applied Mathematics and Informatics, \\ Technical University - Sofia, Bulgaria
}

\begin{abstract}
The aim of this study is to compare the pain intensity reduction between the mean radiation doses per session of gallium-aluminum-arsenide (GaAIAs) laser with superluminous diodes (SLD) in four of the most common pain-related chronic temporomandibular disorders (TMD) - local myalgia, myofascial pain, myofascial pain with a referral, and arthralgia. This study was implemented on 124 patients with pain-related temporomandibular disorders according to the $D C / T M D$ criteria. We applied trigger point oriented near-infrared laser (785 nm, $\left.100 \mathrm{~s}, 8 \mathrm{~J} / \mathrm{cm}^{2}\right)$ and SLD cluster sessions (the cluster is composed of 49 $S L D s$ with a combination of visible red $(633 \mathrm{~nm})$ and infrared $(880 \mathrm{~nm})$ diodes, $200 \mathrm{~mW}$, $300 \mathrm{~s}, 8 \mathrm{~J} / \mathrm{cm}^{2}$ ) for the temporomandibular joints and the affected muscles. Patients were evaluated at the start of the treatment, and after the 6th session of combined phototherapy. The pain intensity scores were measured according to the Visual Analogue Scale (VAS). Our results show that the most statistically manifested pain reduction is found for the SLD dose, $p=0,000118$, followed by the overall dose (laser plus SLD); $p=0,001031$, and the laser dose; $p=0,030942$ (ANOVA dispersion analyses). Consequently, it can be concluded that myalgia is better treated through lower doses of red light compared to infrared laser doses because SLDs combine the prooxidative effect of photons with $633 \mathrm{~nm}$ wavelength, a large area of exposure, sufficient tissue penetration, and some positive warming thermal impact of the SLD clusters.
\end{abstract}

Key words: temporomandibular pain-related disorders, low-level laser therapy, superluminous clusters

Corresponding author: Assoc. Prof. Vasil Sveshtarov, DMD, PhD, Faculty of Dental Medicine, 1 Sv. Georgi Sofiyski st. Sofia, 1431, Tel. + 359888226 863, e-mail: vassilsvechtarov@yahoo.com

\section{INTRODUCTION}

$\mathrm{P}$ hototherapy is defined as a therapeutic physical modality, using photons from the visible and infrared spectrum for tissue healing and pain reduction. Photons are produced in both the visible red and the infrared spectrum $(600-1000 \mathrm{~nm})$ which have been proven to penetrate tissue and to be absorbed at a cellular level. A series of photo- chemical reactions occur resulting in enhanced tissue healing, pain control and stimulation of the vascular, immune and lymphatic systems. It is the only therapeutic modality with a cumulative effect in cells and tissue. Light sensitive chromophores within the cell absorb light energy and initiate a series of important photochemical changes such as increased production of nitric oxide and adenosine triphosphate (ATP), as well as changes in cell membrane perme- 
ability. Electron transfer reactions are highly important in the mitochondrial respiratory chain, where the principal chromophores involved in laser therapy are thought to be situated. The mitochondria and Kreb's Cycle stimulation initiates the production of ATP, providing the cell with the extra energy needed to accelerate the healing process and positively influence pain $[4,16]$.

Phototherapy has been widely employed internationally with noteworthy success. It provides both accelerated tissue healing and pain relief with cumulative effects - an increase in microcirculation resulting in the relief of minor muscle and joint aches, pains and stiffness and relaxation of muscles; for muscle spasms, minor pain and stiffness associated with arthritis. Phototherapy is effective in treating conditions that do not normally respond to traditional therapy interventions. Laser therapy was given the potential of becoming at least twice as effective as non-steroidal anti-inflammatory drugs (NSAIDs), including cyclo-oxygenase-2 inhibitors, if applied with optimal dose and energy [19]. Maia et al. [2] summarize fourteen studies on the use of low-level laser therapy for treatment of TMD from which thirteen show a reduction in pain levels. In nine of them, the pain reduction is registered only in the experimental group, while for the remaining four the effect is observed for both the experimental and the placebo groups. Carrasco et al. [3] applied GaAIAs (780 nm) laser to patients with myofascial pain syndrome and found a significant pain reduction. Fikachkova et al. [6] successfully treated $61 \mathrm{TMD}$ patients applying $10 \mathrm{~J} / \mathrm{cm}^{2}$ and $15 \mathrm{~J} / \mathrm{cm}^{2}$ per session (GaAlAs laser; $400 \mathrm{~mW} ; 830 \mathrm{~nm}$ ) in the areas of painful myofascial trigger points. According to Chang et al.'s [5] meta-analysis on seven articles of the clinical effects of LLLT on TMD pain, the analgesic effect achieved with wavelengths of $830 \mathrm{~nm}$ and $780 \mathrm{~nm}$ is moderate to superior. Other studies have also reported similar results [4]. However, Emshoff et al. [7] argued that laser treatment with a wavelength of $632.8 \mathrm{~nm}$ did not generate the desired analgesic effects.

Phototherapy is a non-thermal modality and can be used when other modalities are contraindicated. The heat generated by the SLD cluster can reach $40^{\circ} \mathrm{C}$ at skin contact after 5 minutes of treatment. The heat generated by the laser cluster can reach $40^{\circ} \mathrm{C}$ at skin contact after 15 minutes of treatment. Some devices combine a red wavelength with near infrared (NIR) wavelength on the basis that the combination of two wavelengths can have additive effects, and can also allow such device type to be more broadly utilized to treat more tissue structures.

Mechanisms of action of red and NIR radiation are associated with activation of electron transfer of mitochondrial respiratory chain. The activation of tissue respiration, along with the increased intensity of metabolism, leads to extremely high accumulation of active forms of oxygen and other radical intermediates. Singlet oxygen is a high-energy form of oxygen, a result of a number of transitions with photons with a wavelength of $632 \mathrm{~nm}$, or significantly less likely with photons with a wavelength of $760 \mathrm{~nm}$. In terms of its chemical reactivity singlet oxygen is far more reactive toward organic compounds. Therefore, 632 $\mathrm{nm}$ wavelength is associated with the generation of singlet oxygen which has a mild pro-oxidative biological effect $[8,9,16,20]$.

Light energy can be produced by a variety of devices such as laser diodes, light emitting diodes (LEDs) or super bright LEDs known as superluminous diodes (SLDs). All these have similar effects at a cellular level. Laser light is well-ordered with synchronized light waves referred to as coherence. Lasers generally produce a focused, pin point of light in higher power density, delivering energy deeper into tissue. Therefore, lasers deliver more targeted light energy to smaller treatment areas. In contrast, SLDs, the newer, medical quality super bright version of LED produces a broader beam of light that is not coherent. The terms super-luminous and superluminescent mean 'amplified spontaneous emission': the emission of fluorescence which experiences significant optical gain within the emitting device. SLDs use a double heterostructure to confine the active region under conditions of high current density, creating a population inversion and enabling amplification of light. This type of light provides a more superficial, even distribution of energy with lower power density and longer treatment time than lasers. SLDs are particularly effective for treating larger areas than lasers such as muscle tension and muscle spasms. Laser cluster and SLD clusters can both be used simultaneously. The laser is used for smaller, deeper target tissue, such as trigger points. Although treating a smaller area, the laser will deliver energy faster than the SLD. The larger SLD cluster covers a broader area of discomfort or is used to decrease muscle tension. The majority of the treatment protocols recommend using both during the treatment sessions. Generally, only one modality is used in each treatment session since the cells, and the clinical condition can be over-stimulated. The selection of device and dose is determined by these consider- 
ations: the amount of skin pigmentation; location and size of the treatment area; depth of the target tissue. Literature supports the use of continuous wave energy for most treatments. The clinician may introduce pulsing if a patient does not respond to a few sessions with continuous wave or if the therapy has reached a plateau [17].

\section{AIM OF THE STUDY}

The aim of this study is to compare the pain intensity reduction according to the radiation dose of gallium-aluminum-arsenide $(785 \mathrm{~nm})$ laser clusters and superluminous diodes (633 nm plus $880 \mathrm{~nm}$ ) in the treatment of four pain-related TMDs [1]: local myalgia, myofascial pain, myofascial pain with a referral, and arthralgia.

\section{MATERIAL AND METHODS}

This study includes 124 patients - 14 men and 110 women, aged between 14-74 years (mean - 40.12; SD - 14.85). Clinical assessments were based on Axis I examination protocol of DC/TMD [1], trigger points were palpated of the lateral pole of the joints and the preauricular areas, masseter, temporal and cervical muscles for confirmation of familiar pain during the function. According to TMJ and myogenic specific conditions treatment protocols (anatomical diagrams, the sites, time and number of joules to be delivered by both the low level laser and the SLD clusters) SLD clusters were positioned over affected muscles (trigger points and taut bands), while simultaneously treating a referred area or trigger point with the hand-held laser device. The hand held laser provides $100 \mathrm{~mW}$ of total power using $3 \times 785 \mathrm{~nm}$ (3-33 mW) infrared low-level laser diodes. The superluminous diode (SLD) arrays deliver up to $500 \mathrm{~mW}$ of infrared and visible red energy to target sites. The SLD cluster is composed of 49 SLDs with a combination of visible red (633 $\mathrm{nm})$ and infrared $(880 \mathrm{~nm})$ diodes. A combination of the laser (100 s, $8 \mathrm{~J} / \mathrm{cm}^{2}$ per spot) and SLD red light sessions $\left(200 \mathrm{~mW}, 300 \mathrm{~s}, 8 \mathrm{~J} / \mathrm{cm}^{2}\right.$ per spot) was applied. The mean overall dose is $33.51 \mathrm{~J}$ per session, the mean laser dose is $21.06 \mathrm{~J}$, and the mean SLD dose is 12.25 .

The pain intensity measurements included visual analog scale (VAS) scores. Dispersion Analysis (ANOVA) was used for statistical analysis. STATISTICA software was utilized.
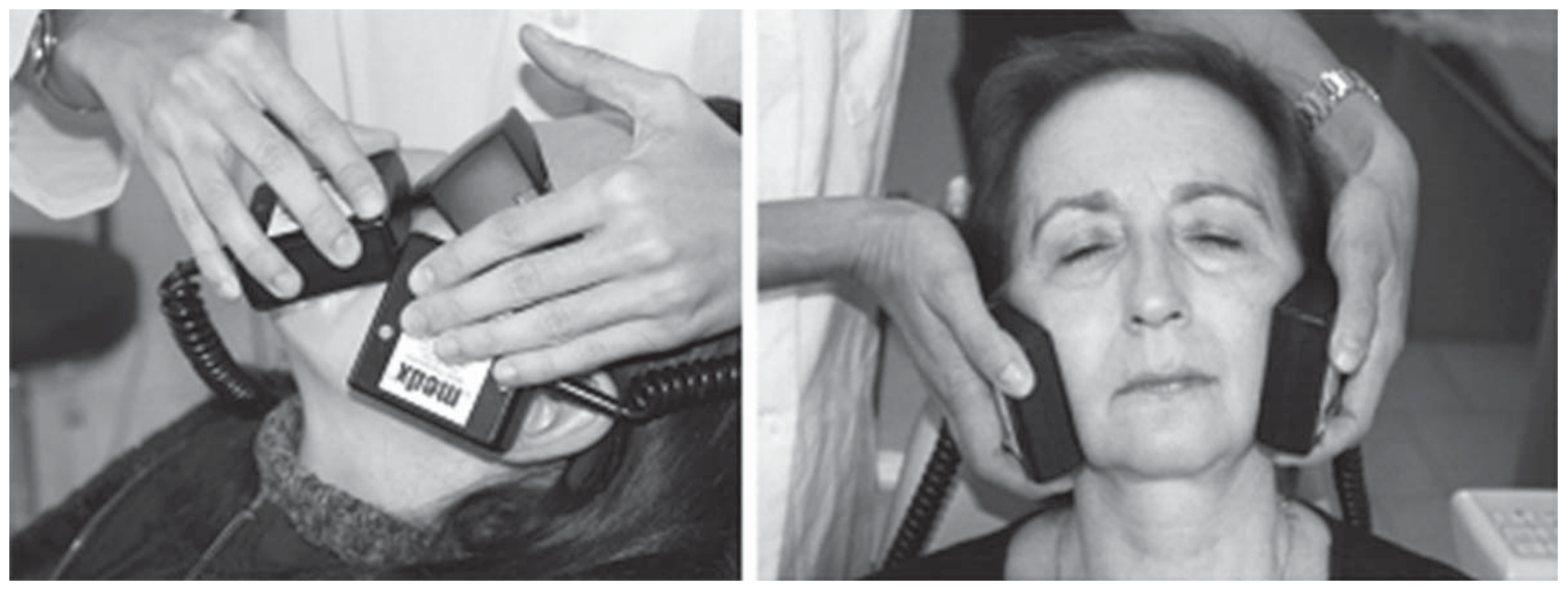

Fig. 1. Clinical application of SLD clusters

\section{RESULTS}

The most statistically manifested pain reduction is found for the mean SLD dose of 12,25 J per session; $p=0,000118$, followed by the mean overall dose of
$33.51 \mathrm{~J}$ per session; $p=0.001031$, and mean laser dose of $21.06 \mathrm{~J}$ per session; $p=0.030942$. The results show that myalgia is best influenced by lower doses of red light from SLD clusters compared to higher infrared laser doses. 
Table 1. Univariate Tests of Significance for mean Laser, SLD, and overall doses per session

Laser dose

Sigma-restricted parameterization Effective hypothesis decomposition

$\begin{array}{llllll} & \text { SS } & \text { Degr. of - Freedom } & \text { MS } & \text { F } & \text { P } \\ \text { Pain reduction in Myalgia and Arthralgia } & 1262,77 & 3 & 420,92 & 3,1694 & 0,030942 \\ & \text { SLD dose } & & & \\ \text { Pain reduction in Myalgia and Arthralgia } & 869,180 & 3 & 289,727 & 8,2426 & 0,000118 \\ & \text { Overall dose } & & & \\ \text { Pain reduction in Myalgia and Arthralgia } & 4070,17 & 3 & 1356,72 & 6,1711 & 0,001031\end{array}$

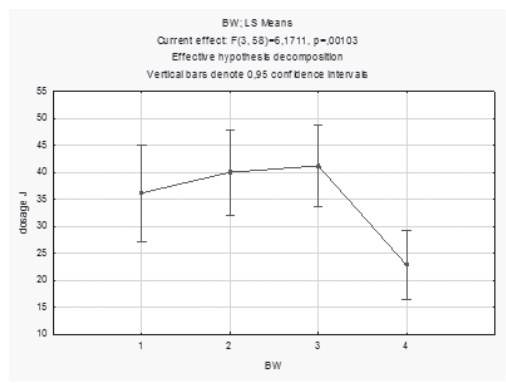

(A - mean overall dose)

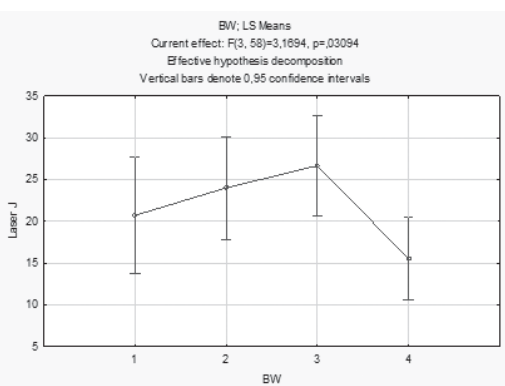

(B - laser dose)

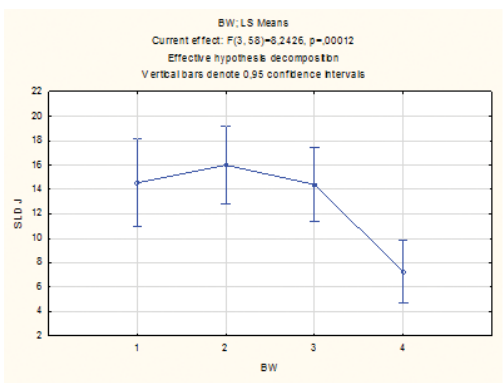

$(\mathrm{C}-\mathrm{SLD}$ dose $)$

Fig. 2. Diagrams of the subgroups distribution for: 1. local myalgia; 2. myofascial pain; 3. myofascial pain with referral; 4. arthralgia
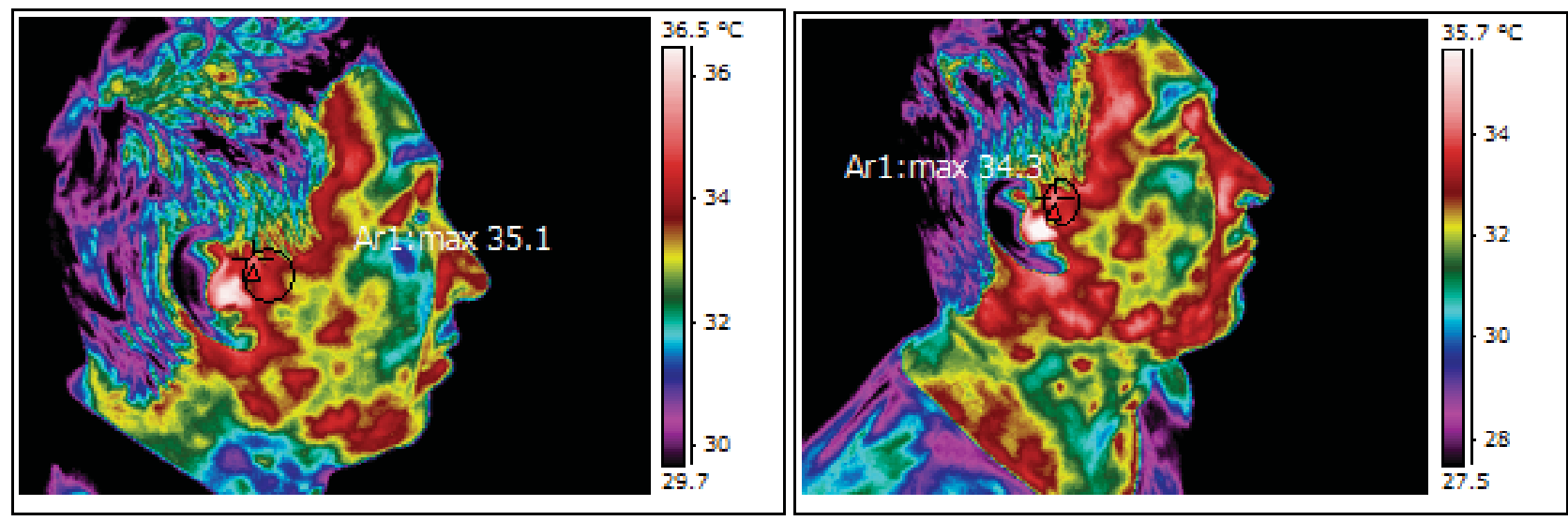

Fig. 3. Thermographic records before and after combined phototherapy show a decrease in joint temperature (from 35.1 to $34.30^{\circ} \mathrm{C}$ ), and visible warming in the masseteric area.

\section{DISCUSSION}

This study shows that combined SLD plus laser phototherapy is very effective particularly in chronic myofascial and temporomandibular pain-related conditions. Unlike most studies that present data for the effect of the single laser therapy on TMJ and masticatory muscles the present investigation provides findings reviewed in the light of the photodevices such as superluminous diodes. At this stage, the comparability of the myogenous
TMD subgroups is hampered by the fact that previous studies have been conducted in accordance with RDC/ TMD of 1992, as well as the relatively rare use of SLDs for TMD therapeutic purposes. The current statistical results show the superiority of SLD diodes in the treatment of the external group of masticatory muscles, and this requires new clinical studies allowing us to refine the picture of the light-source treatment efficiency for TMD in line with the latest views on the subject. One possible explanation for the highly positive clinical effect 
of SLD diodes is the fact that singlet oxygen in the presence of organic-sensitizers molecules is formed as a result of a series of unsolicited transitions in the excitation of triple ungraded levels with photons at a wavelength of $632 \mathrm{~nm}$ or with a much lesser probability by photons with a wavelength of $760 \mathrm{~nm}$. Therefore, irradiation with $632 \mathrm{~nm}$ is associated with the generation of singlet oxygen, and has a mild pro-oxidative biological effect and should be considered as biologically and clinically most effective for superficial tissue structures. Another possible explanation is that lasers deliver more targeted light energy to smaller treatment areas, while myofascial pain is commonly associated with taut bands that require a wider area of light exposure. The larger SLD cluster, which produces visible red light $(600-700 \mathrm{~nm})$, has a superficial penetration of up to $1 \mathrm{~cm}$ and covers a broad area of discomfort, generating a thermal effect. It should be taken into account, that according to Weinstein's TMJ protocol $[10,11]$ for thermographic imaging of the facial area in patients with TMJ syndrome each affected masseter is $1.0^{\circ} \mathrm{C}$ below ambient room temperature; Steed [12] found a $94.5 \%$ sensitivity using thermography for TMJ syndrome, which correlates well with the Weinstein study. Contrary to this Dibai et al. [13] consider that infrared thermography of the masticatory muscles is not an accurate instrument for the myogenous TMD diagnosis. In 2014 Haddad et al. [14] report that the temperature levels measured at the masseter muscle in myogenous TMD volunteers (32.85 \pm 0.85 ) were significantly lower than those measured in controls $(33.49 \pm 0.92$, sensitivity and specificity were of $70 \%$ and $73 \%$ ). On the other hand, Hoffmann et al. [15] based on self-report from a total of 1511 TMJ affected individuals who were recruited through the web-based registry of patients maintained by The TMJ Association, Ltd, among a wide array of treatments used (46 listed), the most effective relief for most affected individuals $(91 \%)$ was the use of thermal therapies-hot/cold packs to the jaw area or hot baths [18]. As mentioned above, the heat generated by the SLD cluster can reach $40^{\circ} \mathrm{C}$ at skin contact after 5 minutes of treatment; the dose of $8 \mathrm{~J} / \mathrm{cm}^{2}$ in continuous mode is realized within 300 seconds. Therefore, the positive thermal effect of the SLD clusters should also be taken into account; so the doses that are used in the red wavelengths for superficial structures rarely exceed $10 \mathrm{~J} / \mathrm{cm}^{2}$.

\section{CONCLUSION}

The present study shows that myogenic temporomandibular disorders are more effectively treated with lower doses of red light delivered by SLD clusters compared to the infrared laser doses. SLD clusters $(633 \mathrm{~nm})$ combine the prooxidative effect of photons with this wavelength, the wider area of exposure, and positive warming thermal effect.

\section{REFERENCES}

1. Diagnostic Criteria for Temporomandibular Disorders (2014), Complete DC/TMD Instrument Set, International RDC/TMD Consortium, http://www.rdc-tmdinternational.org/TMDAssessmentDiagnosis/DCTMD.aspx

2. Maia ML, LR Bonjardin, SS Quintans, et al. Effect of low-level laser therapy on pain levels in patients with temporomandibular disorders: a systematic review. J Appl Oral Sci, 20, 2012, N 6, 594-602.

3. Carrasco TG, LD Gnerisol, DM Gnerisol, MD Mazzeto. Evaluation of low intensity laser therapy in myofascial pain syndrome - Cranio, 27, 2009, N 4, 243-247.

4. Baxter G, C Diamantopoulos, S O'Kane et al. Therapeutic Lasers Theory and Practice. 1997, Churchill Livingstone, New York, 208-209.

5. Chang W, C Lee, $\mathrm{H}$ Lin et al. A Meta-analysis of Clinical Effects of Low-level Laser Therapy on Temporomandibular Joint Pain. J Phys Ther Sci, 26, 2014, N 8, 1297-1300.

6. Fikackova H, T Dostalova, L Navratil et al. Effectiveness of low-level laser therapy in temporomandibular joint disorders: a placebo-controlled study. Photomedicine and Laser Surgery, 25, 2007, N 4, 297-303

7. Emshoff R, Bösch R, Pümpel E, Schöning H, Strobl H. Lowlevel laser therapy for treatment of temporomandibular joint pain: a double-blind and placebo-controlled trial. Oral surgery oral medicine oral pathology oral radiology and endodontics. 105, 2008; N 4, 452-456.

8. Karu T. Primary and secondary mechanisms of action of visible to near-IR radiation on cells. Journal of Photochemistry and Photobiology B: Biology. 49, 1999, N1, 1-17.

9. Hamblin M, Demidov T. Mechanisms of Low Level Light Therapy. Proc. of SPIE; 6140, 2006, 614001.

10. BenEliyahu D. Infrared Thermal Imaging of Temporomandibular Joint Syndrome. Dynamic Chiropractic. 11, 1993, N 02 , January 15, www.dynamicchiropractic.com

11. Weinstein SA. A protocol for the identification of temporomandibular joint disorders by standardized computerized electronic thermography. Clinical Journal of Pain, 3, 1987, N 2:107-112.

12. Steed $P$ : Utilization of contact liquid crystal thermography in the evaluation of temporomandibular dysfunction. Journal of Craniomandibular Practice, 9, 1991, N 2, 121-12.

13. Dibai F et al. Accuracy of Infrared Thermography of the Masticatory Muscles for the Diagnosis of Myogenous Temporomandibular Disorder Journal of Manipulative and Physiological Therapeutics, 36, 2013, N4, 245-252.

14. Haddad DS, Brioschi ML, Vardasca $R$ et al. Thermographic characterization of masticatory muscle regions in volunteers with and without myogenous temporomandibular disorder: preliminary results. Dentomaxillofacial Radiology, 43, 2014, N 8, doi: 10.1259/dmfr.20130440.

15. Hoffmann RG, Kotchen JM, Kotchen TA et al. Temporomandibular disorders and associated clinical comorbidities. Clin J Pain. 27, 2011, N 3, 268-74.

16. Biophysical mechanisms of therapeutic activity of physical factors on organism. www.fmi.uni-sofia.bg/courses/biomed/

17. Baxter D. Low intensity laser therapy. In: Kitchen S, Bazin S, eds. Electrotherapy: Evidence-Based Practice. London: WB Saunders; 2003.

18. In: TMJ Treatments. The TMJ Association, Ltd. (TMJA). www. tmj.org/treatments

19. Tunér J. Laser better than NSAIDS. In: Laser world. Swedish Laser Medical Society. 2005, http://www.laser.nu/index.htm

20. Vladimirov IA, Klebanov GI, Borisenko GG, Osipov AN. Molecular and cellular mechanisms of the low intensity laser radiation effect Biofizika. 49, 2004, N 2, 339-350. 